\title{
NOVEL ORAL DELIVERY OF IBUPROFEN SOLUTION IN HARD GELATIN CAPSULES
}

\author{
FORUM JANAK JALUNDHWALA ${ }^{*}$, VAISHALI LONDHE ${ }^{1}$, JNANADEVA BHAT ${ }^{2}$, SURYA SINGH ${ }^{2}$
}

1Shobhaben Pratapbhai Patel School of Pharmacy and Technology Management, SVKM's NMIMS, Mumbai, India, ${ }^{2}$ Formulation Development Department, ACG Associated Capsules Pvt. Ltd., Mumbai, India

Email: forum.jalundhwala@gmail.com

Received: 04 Mar 2019 Revised and Accepted: 23 Apr 2019

\section{ABSTRACT}

Objective: The primary objective of the project was to formulate and evaluate hard capsule containing the solution of ibuprofen. It also included enhancement of solubility of ibuprofen in hydrophilic solvents to obtain a unit dose capsule acceptable for human consumption.

Methods: Solution of ibuprofen was developed by the salt formation of partial drug using potassium hydroxide in PEG 600 and water. The solution was encapsulated in hard capsules with band sealing. The final formulation was evaluated for uniformity of weight, disintegration, drug content and stability. The dissolution profile was compared with that of available marketed tablets and softgels.

Results: The capsules were evaluated and found compliant as per specifications mentioned in general monograph of capsules in IP 2014. The uniformity of weight of the batch of capsules was found to be $734.8 \mathrm{mg}( \pm 0.58)$. The disintegration time of these capsules was observed to be 4.45 min. The drug content was found to be $100.03 \%$ and the product is stable over three months of test period under room temperature as well as accelerated conditions. The dissolution profile showed that softgels take longer time to release the drug whereas marketed tablets showed a dissolution profile comparable with that of formulated capsules.

Conclusion: The developed capsule is a unit dose of liquid containing solubilized ibuprofen delivering the drug directly into the gastrointestinal tract (GIT). These are newer solid oral dosage forms with higher patient compliance and ease in manufacturing. They require lesser steps and manufacturing area when compared to the manufacturing of compressed tablets.

Keywords: Dissolution, Hard gelatine capsules, Ibuprofen, Ionizing agent, Solubility enhancement

(C) 2019 The Authors. Published by Innovare Academic Sciences Pvt Ltd. This is an open access article under the CC BY license (http://creativecommons.org/licenses/by/4.0/) DOI: http://dx.doi.org/10.22159/ijpps.2019v11i6.32659

\section{INTRODUCTION}

Ibuprofen is a commonly prescribed non-steroidal antiinflammatory drug (NSAID) [1, 2]. It is also widely prescribed for its analgesic properties [3]. The drug is used for the treatment of postoperative, epidural, dysmenorrheal and dental pain in addition to migraine, rheumatoid arthritis and other musculoskeletal disorders [4]. The management of acute pain is satisfactorily achieved on quick absorption of the drug into the bloodstream [5]. The absorption of drug increases when administered directly in the solubilized form [6]. Ibuprofen is also reported to cause gastric disturbances when administered orally $[7,8]$. To minimize this gastric irritation, ibuprofen should be absorbed from the gastrointestinal tract (GIT) as quickly as possible [9]. The solid oral dosage forms are one of the most patient compliant routes of administration. Formulation scientists are working on different methods to decrease the gastric disturbances due to the administration of solid oral dosage forms of ibuprofen. Various researches have tried different formulations like Self Emulsifying Drug delivery systems (SEDDS), Self micro emulsifying drug delivery systems (SMEDDS), solid dispersion, Complexation, cocrystallization and other techniques with aim of increasing bioavailability of ibuprofen when administered orally [10-21]. Many researchers overcome gastric irritation by the use of a targeted drug delivery system. Amongst solid oral dosage forms, coated tablets are most commonly marketed dosage form of ibuprofen [22]. Coated tablets are compressed blend of drug and excipients with a film coating. This process involves many steps and evaluation of many in-process quality control parameters. They also require a large manufacturing area. Any changes in particle size, compression pressure can directly lead to variation in the quality of manufactured tablets [23]. These tablets also are slow in absorption when compared to administration of solubilized ibuprofen.

As a result, lot of latest research includes encapsulation of solubilized liquids containing ibuprofen in softgels. Soft gels are dosage forms manufactured in situ. The shells are made up of gelatine and plasticizer. The liquid to be encapsulated can be a solution, emulsion or a suspension [24]. Softgels require dedicated machines for manufacturing and also poses a large ratio of rejection or quality hurdles during manufacturing. Softgels are also not a very stable dosage form.

Novel approach to overcome these hurdles is to encapsulate solubilized drug into two-piece hard gelatine capsules [25]. The twopiece capsules are available in a variety of material, sizes and shapes. The design of these shells is modified for them to efficiently encapsulate a liquid [26]. They are prefabricated and the liquid fill material is filled. They are then band sealed to avoid any leakage [27]. Such a manufacturing process requires fewer steps and lesser area for large scale production $[28,29]$.

The objective of the study was to formulate and evaluate hard gelatin capsules with ibuprofen solution. It also included a comparative study of the dissolution profile of the formulated hard capsule with marketed softgels and coated tablets.

\section{MATERIALS AND METHODS}

\section{Chemicals and reagents}

Ibuprofen was procured from BASF company (Batch no.: IB1V1517) polyethlene glycol (PEG) 600 from Merck specialities Pvt. Ltd. (Batch No.: SF2S620076) and potassium hydroxide pellets of analytical grade Rankem (Batch No.: P005D05) were procured. Hard gelatine capsules of size 1 (Batch No 1100134276) were provided by ACG Associated Capsules Pvt. Ltd. The de-mineralized (D. M.) water used was prepared in-house. Marketed OTC products of Tablet Brufen ${ }^{\circledR}$ manufactured by Abbott Pvt. Ltd. (batch No. B2867459) and Advil ${ }^{\circledR}$ manufactured by Pfizer (batch no. R13920) were purchased.

\section{Compatibility studies}

Gelatine capsule shells are moisture sensitive. Compatibility studies were conducted between gelatine shells and various solvents including 
water, soya oil, cremophor EL, polysorbate 80, triacetin, propylene glycol, glycerine, PEG 400, PEG 600, Transcutol P, Transcutol P 20\% and Transcutol P 40\%. 10 gelatine capsules of size ' 00 ' were filled upto $80 \%$ with the solvent under test. It was then band sealed using capsule sealing machine (LBS-023 by ACG Pam Pharmaceutical and Allied Machinery Company Pvt. Ltd.) and stored for observation for $7 \mathrm{~d}$. Visual inspection for any cracks or softening of the shell was noted.

\section{Solubility studies}

To study the solubility of ibuprofen, certain solvents were shortlisted from the results of compatibility studies. To test the solubility of drug in individual solvents, $25 \mathrm{ml}$ of each solvent was saturated with the drug. The flasks were subjected to rotary shaking using a shaker for $24 \mathrm{~h}$ at room temperature. The solution was then filtered using whatmann filter paper and diluted with water to obtain absorbance. Absorbance was measured using UV-Visible spectrophotometer (Thermo Scientific Evolution 201) at $221 \mathrm{~nm}$.

\section{Formulation trials}

Depending on the results obtained from solubility and compatibility various formulation trials were conducted as shown in table 1 . Trials were designed to achieve the target fill weight of 700-900 mg for a size ' 00 ' capsule. The drug was dissolved in warm PEG 600. To this, an ionizing agent in the form of potassium hydroxide pellets was added $[30,31]$. It was stirred till homogenous and filled in 10 gelatine capsules of size ' 00 '. It was band sealed and kept under observation. Visual inspection was conducted for any changes in the appearance of gelatine shell.

Table 1: Content (mg/capsule) of ingredients in each formulation trial

\begin{tabular}{|c|c|c|c|c|c|c|c|}
\hline Batch No. & 0001 & 0002 & 0003 & 0004 & 0005 & 0006 & 0007 \\
\hline Ibuprofen & 400 & 400 & 400 & 400 & 400 & 400 & 400 \\
\hline PEG 600 & 400 & 400 & 400 & 250 & 250 & 250 & 225 \\
\hline Potassium hydroxide & 40 & 50 & 50 & 50 & 50 & 50 & 50 \\
\hline Water & 55 & 50 & 100 & 50 & 100 & 80 & 60 \\
\hline
\end{tabular}

\section{Evaluation of formulated capsules}

The liquid blend was prepared as per the developed formula. The batch of liquid was filled in gelatine shells of size '00' as a unit dose of $400 \mathrm{mg}$ and labeled as Batch 0007. The evaluation of capsules included evaluation of the liquid fill material as well as the entire capsule.

\section{Evaluation of liquid fill material}

The liquid fill material of the final formulation was tested for various parameters of the solution.

\section{pH}

The $\mathrm{pH}$ of the fill material was measured using systronics $\mathrm{pH}$ Meter (Universal Enterprise, model no. UD 95)

\section{Viscosity}

The viscosity of fill material was measured using Brookfield viscometer (model DV-II, USA) equipped with spindle S27. The apparent viscosity was measured at room temperature at 50 rotations per minutes (RPM) after five minutes.

\section{Density}

The density of fill material was measured using pycnometer.

\section{Infra-red spectrum}

The Infra Red (IR) spectrum of the formulation was obtained using FTIR instrument (ALPHA-PN10109/03, Bruker, Germany). IR spectrum of the pure drug was also obtained. The characteristic peaks of drug and formulation were compared.

\section{Differential scanning calorimetry (DSC) studies}

The physical status of ibuprofen in the solution was characterized using DSC thermogram analysis (STA 6000 simultaneous thermal analyzer, Perkin Elmer, Waltham, MA, USA). 5 gm of each (ibuprofen and ibuprofen solution) was placed in a standard aluminum pan and then purged with pure dry nitrogen gas at a flow rate of $10 \mathrm{ml} / \mathrm{min}$ prior to analysis. The temperature was increased at a rate of 10 ${ }^{\circ} \mathrm{C} /$ minute and the heat flow was recorded from $30{ }^{\circ} \mathrm{C}$ to $300^{\circ} \mathrm{C}$.

\section{Evaluation of capsules}

The formulated ibuprofen capsules were evaluated as per general monograph of hard capsules in Indian Pharmacopoeia (IP) 2014.

\section{Uniformity of weight}

Variation in weight of each capsule will be proportional to variation in drug content. Thus, uniformity of weight was performed. An intact capsule was weighed. The contents were emptied. The shell was washed with alcohol and weighed. The difference in weight was used to determine the weight of content inside capsule. The same was repeated for 19 more capsules. Average weight and \% RSD were calculated.

\section{Disintegration test}

6 capsules were evaluated for disintegration time using tablet disintegration apparatus. The capsules were inserted using discs in $900 \mathrm{ml} \mathrm{D}$. M. Water at a temperature of $37{ }^{\circ} \mathrm{C}$. The time for its complete disintegration was noted.

\section{Drug content}

10 capsules were opened and contents were emptied. The content equivalent to a unit dose was taken in a $100 \mathrm{ml}$ volumetric flask. To this $20 \mathrm{ml}$ phosphate buffer $\mathrm{pH} 7.2$ (prepared as per IP 2014) was added and sonicated. Volume was made up with phosphate buffer $\mathrm{pH}$ 7.2. The solution was further diluted appropriately. Absorbance were taken using UV-Visible spectrophotometer (Thermo Scientific Evolution 201) at $221 \mathrm{~nm}[32,33]$.

\section{Dissolution studies}

Dissolution studies were conducted in triplicates in USP apparatus 2 (Paddle apparatus). The dissolution media of $900 \mathrm{ml}$ phosphate buffer ( $\mathrm{pH}$ 7.2) was used as per IP 2014. The paddle rotation speed was set at 100 RPM and the temperature as $37^{\circ} \mathrm{C}$. The samples were introduced into the media using sinkers. $10 \mathrm{ml}$ of dissolution sample were withdrawn at definite intervals. The dissolution batch was replaced with fresh media at specified time intervals. The withdrawn samples were filtered through 0.45 microns nylon filters. The samples were appropriately diluted with phosphate buffer $\mathrm{cH}$ 7.2). The absorbance of these diluted solutions was taken at $221 \mathrm{~nm}$ using UV-Visible spectrophotometer.

The dissolution studies were performed on the formulated capsule (batch 0007 labeled as FI001), Advil ${ }^{\circledR}$, a marketed softgel formulation and Brufen ${ }^{\circledR}$, a marketed compressed tablet. The dissolution profiles of three different solid oral dosage forms were statistically compared.

\section{Statistical analysis}

The dissolution profiles of different solid oral dosage forms were compared statistically using unpaired $t$-test (Graph Pad Prism, version 5.0.) [34].

\section{Stability studies}

The samples for stability studies were stored in HDPE containers with canister. The samples were stored at room temperature as well 
as at accelerated conditions at $40{ }^{\circ} \mathrm{C}$ and $75 \%$ Relative Humidity. The capsules were evaluated for stability at intervals of one month, two months and three months.

\section{RESULTS AND DISCUSSION}

\section{Compatibility studies}

Compatibility studies between excipients and hard gelatin capsule shell revealed that the shell collapsed instantly when filled with water. The shell softened after $3 \mathrm{~h}$ in case of propylene glycol or glycerine. The shell showed cracking after $24 \mathrm{~h}$ when filled with PEG 400, PEG 600 or Transcutol P. However, the shell showed no change in its appearance when filled with soyaoil, cremophor EL, Polysorbate 80 , triacetin, transcutol P $20 \%$ or transcutol P $40 \%$.
Since the main objective is to formulate an aqueous solution, hydrophilic solvents were majorly considered for formulation development. Water, PEG 600 and PEG 400 were tested as solvents. However, compatibility results proved that the hydrophilic solvents cannot be used alone. They either lead to cracking or softening of capsule shells. Thus, Propylene Glycol and Glycerol were also tested for solubility as they can work as a co-solvent as well as plasticizer. Other components tested for compatibility majorly play a role in drug release and not solubility. Hence, they were not tested for solubility studies.

\section{Solubility studies}

The solubility of ibuprofen in every solvent under test was calculated and results were noted (fig. 1).

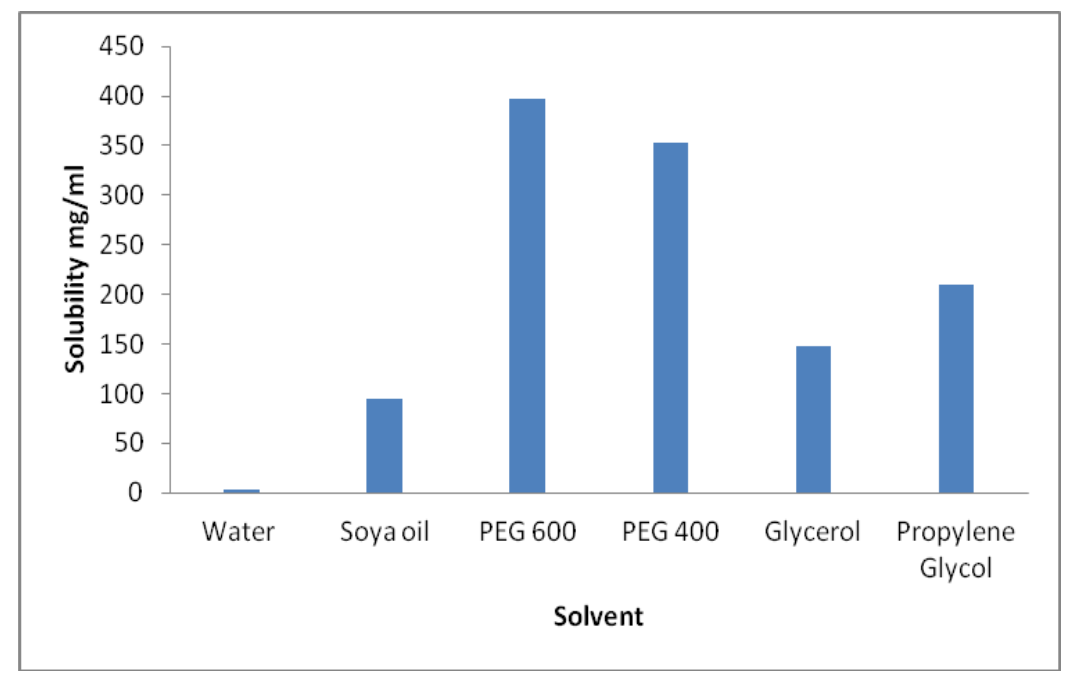

Fig. 1: Solubility of Ibuprofen in test solvents

The solubility was found maximum in PEG 600. Compatibility results indicated that $100 \%$ hydrophilic solvents cannot be used. Capsules being a unit dosage form entire dose should be incorporated in one capsule whose size is acceptable for human consumption. This led to trials of different methods for solubility enhancement.

The common approaches for solubility enhancement are solid dispersion, particle size reduction, complexation, $\mathrm{pH}$ adjustment, salt formation to name a few. Ibuprofen procured was already micronized and so size reduction was not tried. Solid dispersion and complexation requires polymer in atleast 1:1 ratio. With $400 \mathrm{mg}$ of dose, such methods would increase the target fill weight for a standard size of capsule for human consumption. On the basis of a literature search trials were focused on salt formation mechanism to increase solubility and achieve solution of unit dose in a consumable capsule.

\section{Formulation development}

Capsules are unit dosage forms and thus the entire dose of drug needs to be incorporated in one capsule. Size 00 can approximately accommodate $850 \mathrm{mg}$. It can vary to some extent depending on the density. The objective thus remains to solubilize $400 \mathrm{mg}$ of ibuprofen to make final formulation of not more than $850 \mathrm{mg}$.

As shown in table 1 formulation trails were designed using ionizing agent (Potassium hydroxide pellets) to enhance solubility. Batch 0001 did not show complete solubility of drug probably due to insufficient alkaline environment for solubilization. In the next formulation trial batch 0002 concentration of potassium hydroxide pellets were increased to obtain complete solubility. But it lead to cracking within $24 \mathrm{~h}$ of filling and sealing. This was thought to be due to presence of unreacted hydroxyl ions or due to hygroscopic nature of ionizing agent. To overcome this, a humectant in the form of increased water content was explored. Thus in batch 0003 concentration of water was increased. The capsules of this batch were also found to crack within $24 \mathrm{~h}$. This indicated that the water added is not enough to counterbalance the hygroscopicity of formulation. This behavior is predominantly due to hygroscopic nature of either PEG 600 and/or ionizing agent. In Batch 0004 concentration of PEG 600 was reduced keeping concentration of water enough to solubilize the drug. The capsules of this batch also showed cracking within $24 \mathrm{~h}$ of filling and sealing. Further increase in concentration of water was tried. Batch 0005 contained an increased concentration of water. This batch showed softening of capsule shells within $24 \mathrm{~h}$ of filling and sealing. This indicated that decreased concentration of PEG 600 with increase in concentration of water with optimum level can make formulation compatible with hard capsule shell. Batch 0006 contained decreased concentration of water as compared to batch 0005 . The capsules of this batch also showed softening after $3 \mathrm{~d}$ of filling and sealing. As next trial the concentration of water was further reduced. Batch 0007 with reduced water was stable and compatible.

\section{Evaluation of formulated capsules}

\section{Evaluation of liquid fill material}

The liquid of ibuprofen was tested for various parameters. The $\mathrm{pH}$ of the solution was found to be 6.69 . The viscosity of the solution was $441.9 \mathrm{cps}$ and the density of the solution was $1.07 \mathrm{~g} / \mathrm{ml}$.

\section{Infra-red spectrum}

The IR spectrum of pure ibuprofen and the liquid formulation was recorded (fig. 2). Ibuprofen has its characteristic peak of carbonyl group near $1700 \mathrm{~cm}^{-1}$. The spectrum indicates that the characteristic peaks of pure drugs remain in the liquid. Thus, the drug is not degraded. 


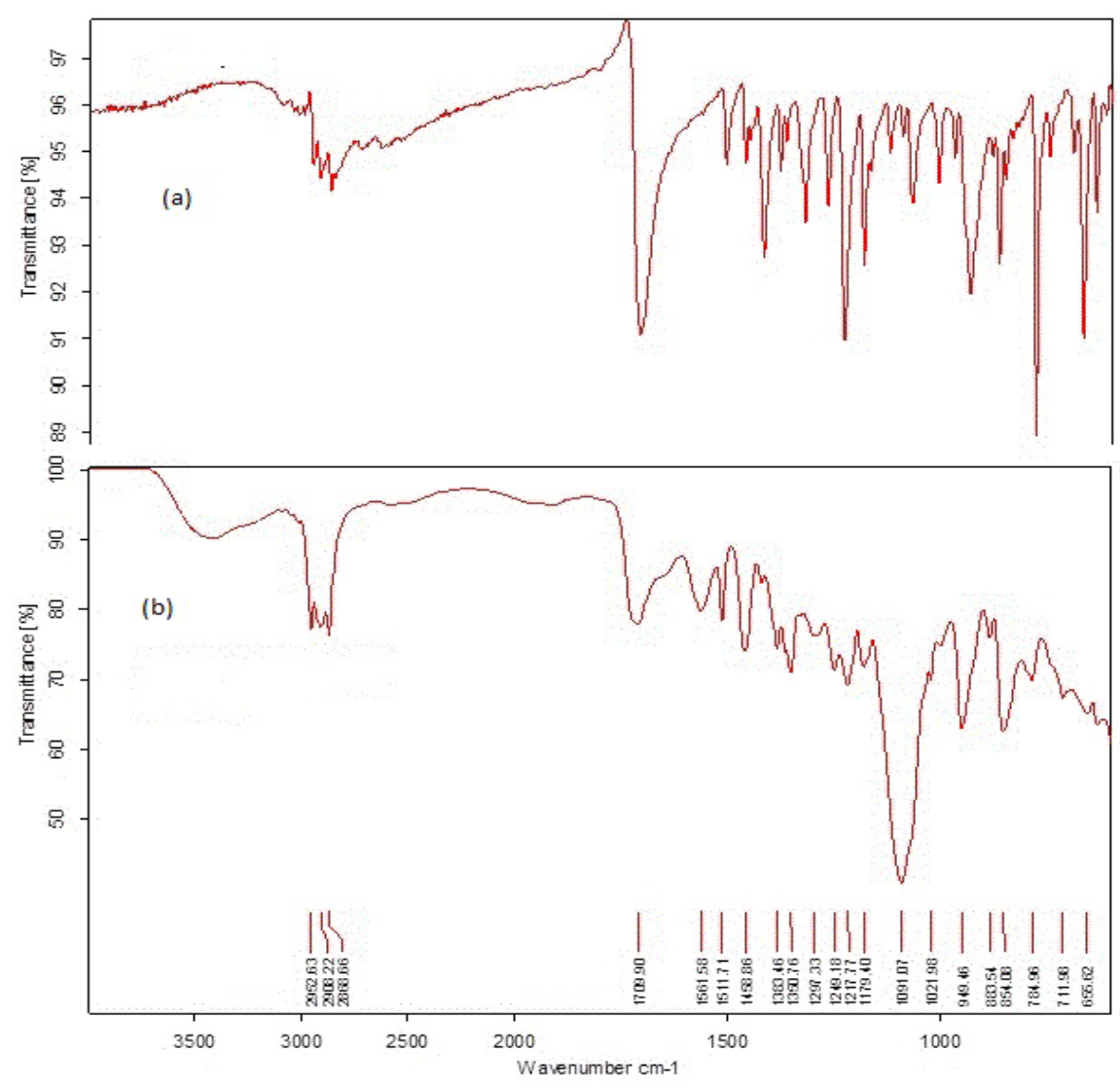

Fig. 2: IR spectrum of (a) pure ibuprofen and (b) formulation of ibuprofen
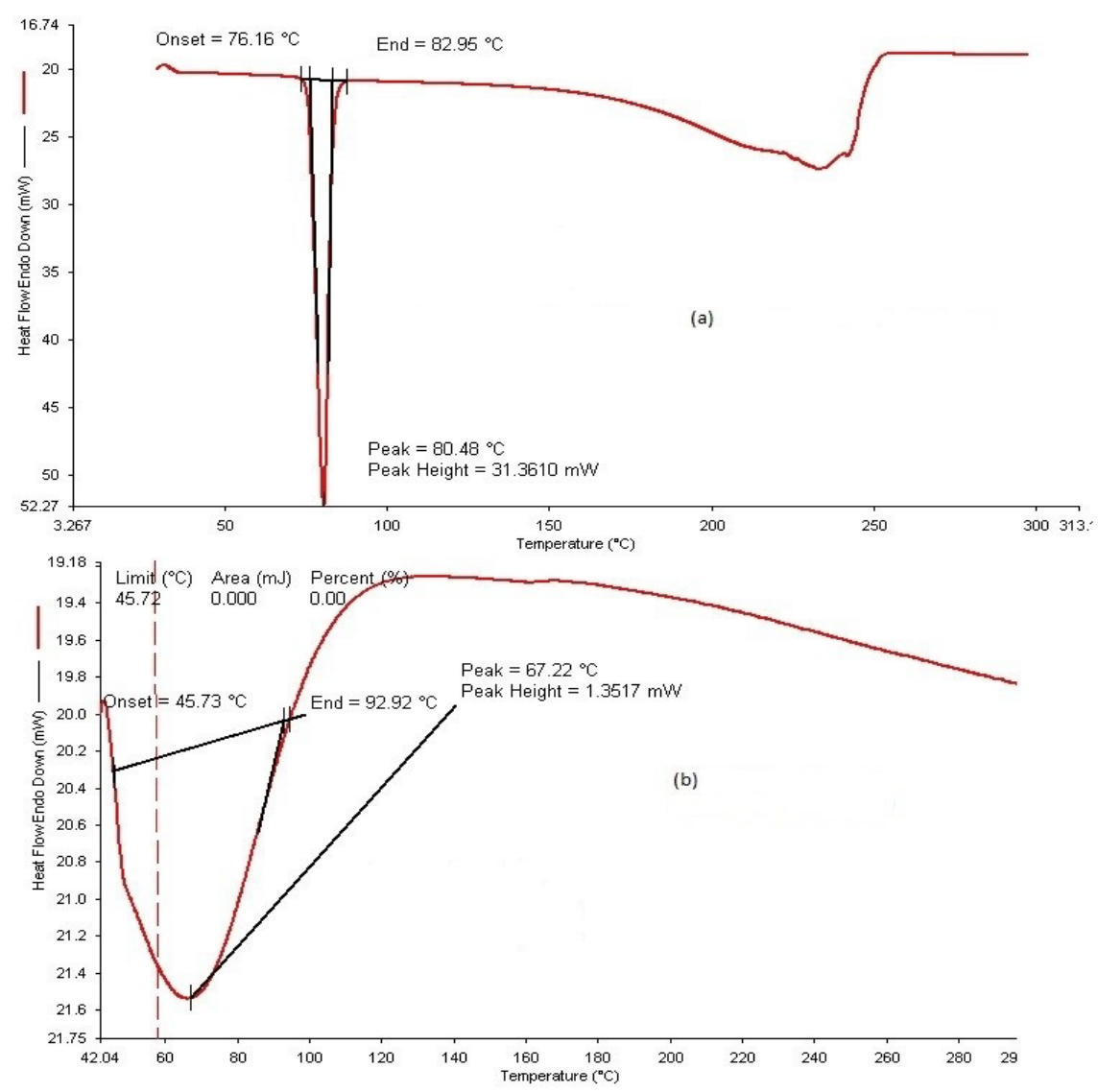

Fig. 3: DSC thermogram of (a) ibuprofen and (b) formulation of ibuprofen 


\section{Differential scanning calorimetry (DSC) studies}

The results from DSC studies were recorded (fig. 3).

The DSC studies measures melting points of drug and excipients. The melting point of drug changes with its crystalline nature. If the drug remains crystalline in formulation then no change in peak would be observed. The sharp peak in fig. above indicates melting point. The peak of pure ibuprofen is observed at $80.48{ }^{\circ} \mathrm{C}$. Broad endotherm is seen which may be due to presence of water. The peak of pure ibuprofen is merged in a formulation that shows solubilization of drug.

\section{Evaluation of capsules}

The capsules were evaluated and found compliant as per specifications mentioned in general monograph of capsules in IP 2014. The uniformity of weight of the batch of capsules was found to be $734.8 \mathrm{mg}( \pm 0.58)$. The disintegration time of these capsules was observed to be $4.45 \mathrm{~min}$. The drug content was found to be $100.03 \%$.

\section{Dissolution Studies}

Dissolution profile is graphically represented (fig. 4).

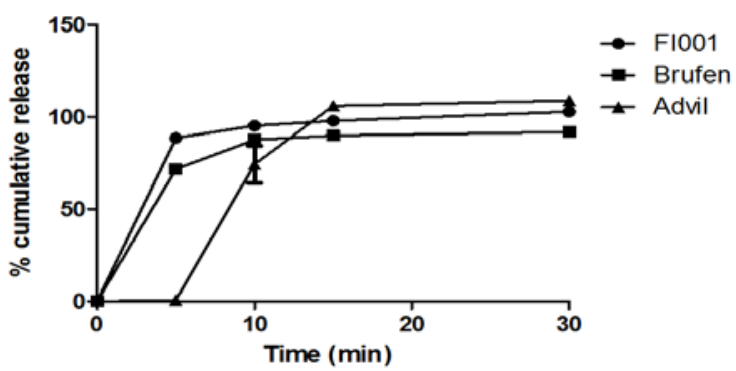

Fig. 4: Dissolution profile in phosphate buffer $\mathrm{pH} 7.2($ mean \pm SD of $n=3$ )

The dissolution profile shows that softgels (Advil ${ }^{\circledR}$ ) take longer time to release the drug. This is thought due to the longer disintegration time of softgels. The walls of softgels are thick and hence it may take a longer time to open and release the solubilized drug. The tablets are marketed as immediate release dosage forms and show a dissolution profile comparable with that for capsules of FI001 (formulated capsules from trial batch 0007). The dissolution results of softgels and tablets were compared with formulated capsules statistically using unpaired $t$-test. The $\mathrm{p}$ values with Advil ${ }^{\circledR}$ as well as Brufen ${ }^{\circledR}$ were found to be $>0.05$ and thus there was no significant difference between the dissolution of different dosage forms.

\section{Stability studies}

The capsules were visually inspected for stability at timely intervals. The visual inspection included observation for any leakage or change in texture of capsule shell.

The dissolution profiles obtained on intervals of $1 \mathrm{mo}, 2 \mathrm{mo}$ and 3 mo were compared with that of initial dissolution profile. The samples were stored at different conditions and are graphically presented (fig. 5).

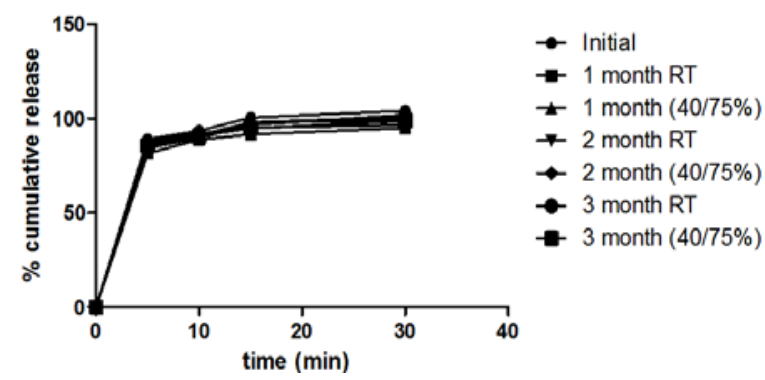

Fig. 5: Dissolution profiles of stability samples (mean \pm SD of $n=3$ )
The dissolution profiles of stability samples showed that the product is stable over three months of test period under room temperature as well as accelerated conditions.

\section{CONCLUSION}

The developed capsule is a unit dose of liquid containing solubilized ibuprofen. The liquid is encapsulated in hard gelatine capsules with band sealing to avoid leakage. These capsules deliver drug directly in solubilized state in GIT. The hard capsules were found to deliver drug as an immediate delivery system. This increases bioavailability and decreases gastrointestinal disturbances caused by ibuprofen. The dissolution profile of these capsules showed higher release as compared to marketed softgels and tablets at the end of $5 \mathrm{~min}$. These capsules may be suggested to have a more immediate effect as compared to other forms of immediate release. Manufacturing of these capsules is easier as there are less number of steps when compared to manufacturing of compressed tablets. The in-process quality control checks are less and variation is also minimal. Thus they have fewer rejections as compared to manufacturing of softgels. These are newer solid oral dosage forms with higher patient compliance and ease in manufacturing along with economic benefits.

\section{AUTHORS CONTRIBUTIONS}

The study was initiated and the protocol was designed by Jnanadeva Bhat. All experimental work was conducted by Forum Jalundhwala. The work was monitored by Vaishali Londhe and Surya Singh. Manuscript was written by Forum Jalundhwala under the guidance of Vaishali Londhe.

\section{CONFLICT OF INTERESTS}

All authors have non to declare

\section{REFERENCES}

1. Rabia Bushra Mhs, Nousheen Aslam, Masud-Ur-Rehman Dha Formulation development and optimization of ibuprofen tablets by direct compression method. Pak J Pharm Sci 2008;21:113-20.

2. Abraham P, KI KD. Nitro-arginine methyl ester, a non-selective inhibitor of nitric oxide synthase reduces ibuprofen-induced gastric mucosal injury in the rat. J Dig Dis 2005;50:1632-40.

3. Wood DM, Monaghan J, Street P, Jones AL, Dargan PI. Fatality after deliberate ingestion of sustained-release ibuprofen; a case report. Crit Care 2006;10:R44.

4. Potthast $\mathrm{H}$, Dressman JB, Junginger HE, Midha KK, Oeser $\mathrm{H}$, Shah VP, et al. Biowaiver monographs for immediate release solid oral dosage forms: ibuprofen. J Pharm Sci 2005;94:212131.

5. Lodha A Patel, J Dalal. Formulation and evaluation of transparent ibuprofen soft gelatine capsule. J Pharm Bioallied Sci 2012;4:S95-97.

6. Rinkesh M Patel, Hema N Patel, Dhaval G Gajjar, Pravinkumar M Patel. Enhanced solubility of non-steroidal antiinflammatory drugs by hydroxyl terminated s-triazine based dendrimers. Asian J Pharm Clin Res 2014;7:156-61.

7. Linghui Dian, Zhiwen Yang, Li F. Cubic phase nanoparticles for sustained release of ibuprofen: formulation, characterization, and enhanced bioavailability study. Int J Nanomed 2013;8:84554.

8. PK Lakshmi, Marka Kranthi Kumar, Aishwarya Sridharan, Shyamala Bhaskaran. Formulation and evaluation of ibuprofen topical gel: a novel approach for penetration enhancement. Int J Appl Pharm 2011;3:25-30.

9. Shyamala Bhaskaran, Pradeep GC, Lakshmi PK. Formulation and evaluation of diphenhydramine hydrochloride and ibuprofen soft gelatine capsules. J Appl Pharm Sci 2011;1:18890.

10. Rubendra Kurmi, Dinesh Kumar Mishra, Dinesh Kumar Jain. Solid dispersion: a novel means of solubility enhancement. J Crit Rev 2016;3:1-8.

11. Murtha JL, Ando HY. Synthesis of the cholesteryl ester prodrugs cholesteryl ibuprofen and cholesteryl flufenamate and their formulation into phospholipids microemulsions. J Pharm Sci 1994;83:1222-8. 
12. Abbaspour MR, Sadeghib F, Garekani HA. Design and study of ibuprofen disintegrating sustained-release tablets comprising coated pellets. Eur J Pharm Biopharm 2008;68:747-59.

13. Abdelazim Zaghloul Ana, Ibrahim Khattab. Development, characterization and optimization of ibuprofen self-emulsifying drug delivery system applying face centered experimental design. Int J Pharm Technol 2011;3:1674-93.

14. Bharat Bhushan Subudhi, Surjyanarayan Mandal. Selfmicroemulsifying drug delivery system: formulation and study intestinal permeability of ibuprofen in rats. J Pharm Sci 2013;6. DOI:10.1155/2013/328769

15. M Saquib Hasnain, Amit Kumar Nayak, Bulgarian. Solubility and dissolution enhancement of ibuprofen by solid dispersion technique using peg 6000-Pvp K 30 combination carrier. Bulgarian J Sci Education 2012;21:118-32.

16. Shravan Kumar Patel, Dinesh Kumar, Amol P, Waghmod, Avinash S Dhabale. Solubility enhancement of ibuprofen using hydrotropic agents. Int J Pharm Life Sci 2011;2:542-5.

17. Tapan Kumar Giri, Hemant Badwaik, Amit Alexander, Dulal Krishna Tripathi. Solubility enhancement of ibuprofen in the presence of hydrophilic polymer and surfactant. Int J Appl Biol Pharm Technol 2010;1:793-800.

18. Amit Chaudhary UN, Neha Gulati, VK Sharma, RL Khosa. Enhancement of solubilization and bioavailability of poorly soluble drugs by physical and chemical modifications: a recent review. J Adv Pharm Edu Res 2012;2:2249-3379.

19. Monzurul Amin Roni, Reza-Ul Jalil. Comparative study of ibuprofen solubility in synthetic and natural lipid vehicles. DUJPS 2011;10:65-6.

20. Varun Raj, Vemula VL, Srikanth Lingala. Solubility enhancement techniques. Int J Pharm Sci Rev Res 2010;5:41-51.

21. Arif Budiman, Elis Nurlatifah, Saeful Amin. Enhancement of solubility and dissolution rate of glibenclamide by cocrystal approach with solvent drop grinding method. Int J Curr Pharm Res 2016;7:248-50.

22. Manfred Lahner, Klaus Posselt. Ibuprofen-containing soft gelatine capsules and process for preparing same. US Patent 4,690,823; 1987.
23. Rasenack N, Muller BW. Ibuprofen crystals with optimized properties. Int J Pham 2002;245:9-24.

24. Harsha Kathpalia, KS Gaurav Doshi. Recent trends in hard gelatine capsule delivery system. J Adv Pharm Edu Res 2014;4:165-77.

25. Martin Kuentz, Dieter Rothlisberger. Determination of the optimal amount of water in liquid-fill masses for hard gelatine capsules by means of texture analysis and experimental design. Int J Pharm 2002;236:145-52.

26. Joseph A Pace, Two Piece Hardshell. Soluble and digestible liquid containing gelatine capsule. U. S. Patent 4,281,763; 1981.

27. Ewart T. COLE liquid filled and sealed hard gelatine capsules. Capsugel Library 1986;12:11-3.

28. Stephen Brown. Liquid-fill based formulation: advances and challenges. Ingredients, Formulation and Finishing; 2004. p. 64-8.

29. Dennis. Two-piece hard capsules for pharmaceutical formulations. Pharmaceutical Processes; 2013.

30. Man SYu, Rochester NY Foo. Solvent system enhancing the solubility of pharmaceuticals for encapsulation. U. S. Patent 5,071,643; 1991.

31. Shyamala Bhaskaran. Formulation and evaluation of diphenhydramine hydrochloride and Ibuprofen soft gelatine capsules. J Appl Pharm Sci 2011;1:188-90.

32. Riddhi Gondalia RM, Pankaj Savaliya. Development and validation of spectrophotometric methods for simultaneous estimation of ibuprofen and paracetamol in soft gelatine capsule by simultaneous equation method. Int J Chemtech Res 2010;2:1881-5.

33. Zareena Yasmeen Tm, Heena Farheen, Husna Kanwal Qureshi. Dissolution method development and validation for combination of ibuprofen and paracetamol tablets. Asian J Pharm Clin Res 2013;6:164-8.

34. Nilufer Yuksel A, Arzu E, Kanık B, Tamer Baykara. Comparison of in vitro dissolution profiles by ANOVA-based, modeldependent and-independent methods. Int J Pharm 2000;209:57-67. 